\title{
Electron field emission from surfaces with steps
}

\author{
I. Merrick and J. E. Inglesfield \\ School of Physics and Astronomy, Cardiff University, Cardiff, CF24 3YB, Wales, United Kingdom \\ G. A. Attard \\ School of Chemistry, Cardiff University, Cardiff, CF10 3TB, Wales, United Kingdom \\ (Received 23 February 2005; published 6 July 2005)
}

\begin{abstract}
First-principles electron field emission calculations have been performed on flat and stepped Pt and Pd surfaces. An increase in electron transmission is seen for well-defined stepped surfaces. This stems from the reduction in the work function caused by Smoluchowski electron-smoothing and an increased tunneling contribution from surface parallel wave vectors at the stepped surface. A reduced effective potential at the step site may also contribute to increased electron transmission.
\end{abstract}

DOI: 10.1103/PhysRevB.72.033403

PACS number(s): 79.70.+q, 73.20.-r, 73.40.-c

Field emission from surfaces has recently received renewed attention because of modern device applications. For instance, polyatomic protrusions from surfaces, such as Spindt-type emitters and carbon nanotubes, are of particular interest because of their use in flat panel displays ${ }^{1,2}$ and vacuum microelectronic devices. ${ }^{3}$ In all cases the current density of tunneling electrons is strongly dependent on the material, its work function, and the external field at the surface. Field emission is, of course, an old subject, and early evidence of surface states came from field-emission experiments on W(001). ${ }^{4}$ The theory of field emission similarly has a long history: the Fowler-Nordheim theory, based on a simplified surface potential, leads to the famous FowlerNordheim plot of $\ln \left(J / F^{2}\right)$ against $1 / F$, where $J$ is the current and $F$ is the applied field. This generally shows a straight line for a small range of $F$. There remain relatively few absolute calculations of $J$ using first-principles methods, ${ }^{5}$ despite the continual experimental interest. This interest is mainly based on increasing emitter functionality, such as field enhancing ${ }^{6}$ at the surface via Spindt-type emitters or carbon nanotube modified surfaces-both of which involve high curvature tips to increase the effects of the external field at the surface..$^{1,2,7-9}$ Other work involves the reduction of the emitter work function via material coatings or annealing in order to reduce the barrier to electron transport. ${ }^{10-12}$

This paper uses a different and accurate method for calculating field emission from flat and stepped $\mathrm{Pd}$ and Pt surfaces. We shall consider the role of work function changes in modifying emission. These effects at stepped surfaces contain the essential ingredients needed to increase emitter functionality.

The calculations are based on the embedding method, ${ }^{13}$ using codes developed by Ishida and his collaborators, ${ }^{14}$ based on the Landauer-Büttiker formalism ${ }^{15}$ for tunneling transmission. The code first implements a self-consistent calculation of the electronic structure of the surface in a field, using full-potential linearized-augmented plane waves (FLAPW). The tunneling current is found from a Green's function expression, reformulated in the embedding scheme. ${ }^{16,5}$ The implementation only takes into account the current from extended bulk states, and the current from sur- face states is neglected. This is because lifetime effects are ignored, in which case the surface states are not in equilibrium with the semi-infinite electron gas. Note that the current from surface resonances is included, so we are only neglecting states from band gaps. Fortunately, for the metal surfaces that we study here, surface states are mainly confined to unbound energies, ${ }^{17-20}$ and their role can be ignored. This is quite different from the (111) surfaces of other noble metals, where emission from the surface state that crosses the Fermi energy dominates. ${ }^{21}$

The embedding method is a variational technique that allows us to concentrate on the surface region (region I) for the self-consistent procedure and the field emission. This region is embedded onto a bulk substrate (region II) with an embedding potential $G_{S}^{-1}$ defined on the boundary $S$ between the two regions. The embedding potential is the surface inverse of a substrate Green's function over $S$ and, added onto the Hamiltonian for region I, it ensures that the wave functions in region I match onto the substrate. ${ }^{13}$ This enables us to solve the Schrödinger equation explicitly only in the region of interest. In the case of surfaces this consists of the top few layers of the metal and the near-surface region extending into the vacuum.

To calculate the field emission, we start from the wellknown formula for the total transmission across some barrier region, ${ }^{22}$ embedded onto semi-infinite reservoirs on each side

$$
T(E)=4 \operatorname{Tr}\left[G(1,2) \operatorname{Im} G_{S_{2}}^{-1}(2,2) G^{*}(2,1) \operatorname{Im} G_{S_{1}}^{-1}(1,1)\right] .
$$

Here $G$ is the Green's function across the region through which transmission is taking place, and $G_{S}^{-1}$ is the embedding potential, embedding this region onto the substrates over surface 1 or 2 . In the original literature on transmission, $G_{S}^{-1}$ is described as the self-energy, and Wortmann et al. ${ }^{16}$ realized that this is the same as the embedding potential. To apply this to the field emission problem, surface 1 is the embedding plane used in the surface calculation, that is, a plane one or two layers below the surface, beyond which the potential is essentially bulklike. Transmission is through the surface layers and the near-surface region with the applied field, to a 
TABLE I. Bulk state transmission current densities $\left(\times 10^{8} \mathrm{~A} / \mathrm{m}^{2}\right)$ with an applied field of $0.65 \mathrm{~V} / \AA$, and work function values $(\mathrm{eV})$ obtained from the embedding code for different surfaces of Pt and Pd.

\begin{tabular}{ccccc}
\hline \hline & \multicolumn{2}{c}{$\mathrm{Pt}$} & \multicolumn{2}{c}{$\mathrm{Pd}$} \\
\cline { 2 - 5 } & $J$ & $\Phi$ & $J$ & $\Phi$ \\
\hline$(111)$ & 0.04 & 6.01 & 0.53 & 5.86 \\
$(001)$ & 0.11 & 6.07 & 1.10 & 5.80 \\
$(311)$ & & & 1.70 & 5.64 \\
$6 \times(001)$ stepped $^{\mathrm{a}}$ & 0.49 & 5.93 & 4.45 & 5.50 \\
\hline \hline
\end{tabular}

Island structure representation.

plane in the asymptotic region of the field. At this plane the embedding potential is calculated from the Green's function in the uniform field.

The tunneling current can be formulated in terms of the transmission, and Ohwaki et al. ${ }^{5}$ have shown that the current density $J$ is given by

$$
\begin{aligned}
J= & \int_{\mathrm{SBZ}} d k_{\|} \frac{2}{(2 \pi)^{2}} \int_{-\infty}^{E_{F}} d \epsilon \frac{2 e}{\pi \hbar} \times \int_{S_{1}} d S_{1} d S_{1}^{\prime} \int_{S_{2}} d S_{2} d S_{2}^{\prime} G(1,2) \\
& \times \operatorname{Im} G_{S_{2}}^{-1}\left(2,2^{\prime}\right) G^{*}\left(2^{\prime}, 1^{\prime}\right) \operatorname{Im} G_{S_{1}}^{-1}\left(1^{\prime}, 1\right) .
\end{aligned}
$$

The first integral is of the surface parallel wave vector $k_{\|}$ over the surface Brillouin zone, denoted by SBZ; the second integral is over energies up to the Fermi energy, starting from some energy below the bottom of the band. The inner integrals are over the two embedding planes, in the metal and vacuum.

Using this transmission technique, we present electron emission calculations for flat $\operatorname{Pd}(001), \operatorname{Pt}(001)$, and $\operatorname{Pt}(111)$ surfaces, as well as stepped surfaces of Pt and Pd involving (001) terrace and (111) step facets. There are practical problems in the present implementation of the embedding method for treating stepped surfaces of low step density arising from the small interlayer spacing and large surface unit cell. To avoid such difficulties, for stepped surfaces with a step density less than that of a face-centered-cubic (fcc) (311) surface, we adopt a superstructure geometry, comprising onedimensional islands placed on top of a low-index substrate. A vicinal fcc (511) surface is therefore replaced in our scheme by a $6 \times(001)$ supercell with a three-atom-wide island placed on top. This scheme then mimics the terrace and step geometry of the fcc (511) surface and also the step density. For the (311) surfaces, methodology and computational resources allow us to use truly vicinal geometries. In all cases, bulk-terminated positions are used for the surface atoms. The accuracy of such structures is dealt with in separate work. ${ }^{23}$

The field emission from the different surfaces is presented in Table I, with an external field of $0.65 \mathrm{~V} / \AA$ applied normal to the surface. Also shown in this table are the calculated values of the work function $\Phi$. Comparing first of all the low-index surfaces, we see that field emission from $\mathrm{Pd}$ is a factor of 10 greater than from Pt. The work function differ-
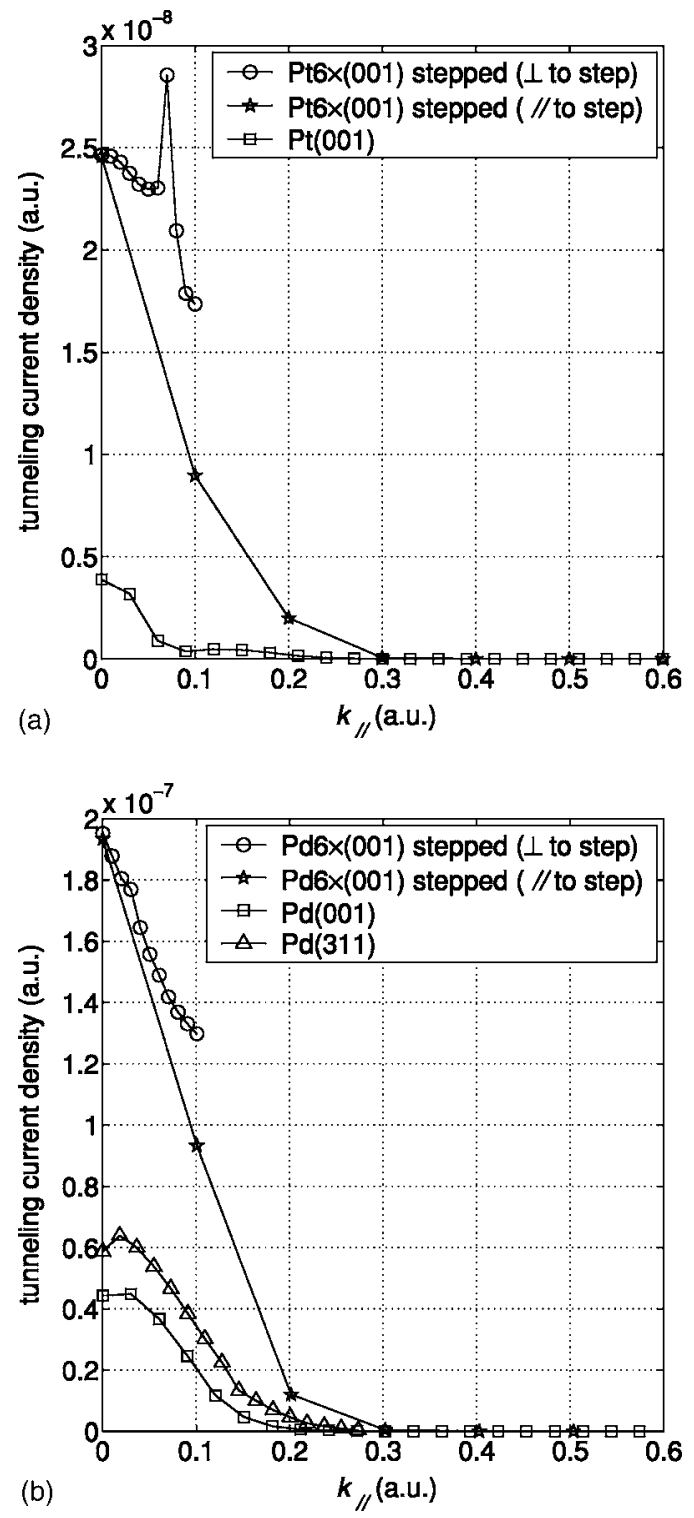

FIG. 1. Tunneling current density as a function of surfaceparallel wave vector $k_{\|}$for (a) $\operatorname{Pt}(001)$ ( $\left.\boxminus\right)$ and stepped Pt6 $\times(001)$ [parallel $(\star)$ and perpendicular $(\ominus)$ to the step face] surfaces, and (b) $\operatorname{Pd}(001)(\boxminus), \operatorname{Pd}(311)(\triangle)$, and $\operatorname{Pd} 6 \times(001)$ [parallel $(\star)$ and perpendicular $(\ominus)$ to the step face]. In each case the wave vector $k_{\|}$extends to the edge of the surface Brillouin zone.

ence presumably plays a role here, with the $\operatorname{Pd}(001)$ work function being $0.25 \mathrm{eV}$ less than that of $\mathrm{Pt}(001)$ for example. However, there must be major material effects at work because there is a much larger reduction in $\Phi$ in going from $\mathrm{Au}(001)$ to $\mathrm{Cu}(001), 0.66 \mathrm{eV}$, and the increase in calculated field emission is a factor of $15,{ }^{5}$ not so much greater than in going from Pd to Pt. For both metals, the more open (001) surface gives greater field emission than the close-packed (111) surface. This cannot be associated with changes in the work function, which is greater for $\operatorname{Pt}(001)$ than for $\operatorname{Pt}(111)$, and less for $\operatorname{Pd}(001)$ than for $\operatorname{Pd}(111)$.

Surface steps lead to an increase in electron emission, with a fourfold increase for both $\mathrm{Pd}$ and $\mathrm{Pt}$ in going from (001) to the $6 \times(001)$ stepped surface. In both cases this is 


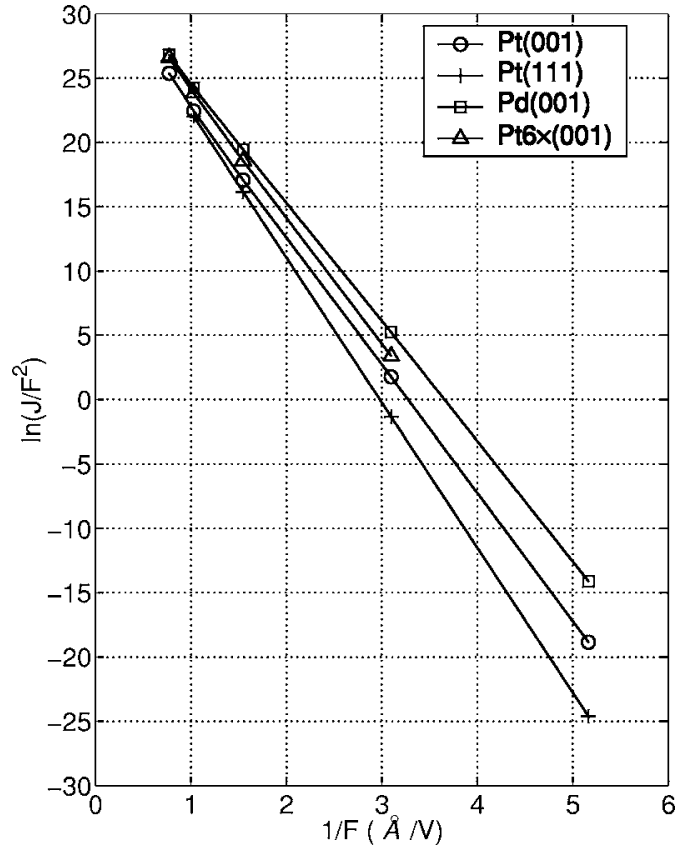

FIG. 2. Fowler-Nordheim plot of emission current densities for Pt(001), Pt(111), Pt6×(001) (stepped), and Pd(111) surfaces. In all cases only emission from bulk states are reported. Current density $J$ is in amperes per meters squared external field $F$ is in volts per angstrom.

associated with a reduction in $\Phi$, but the reduction is only $0.14 \mathrm{eV}$ for Pt. Perhaps more important is the variation in the local work function near the surface steps themselves, ${ }^{23,24}$ which is much bigger than the change in $\Phi$ itself. Here we define the local work function as the difference in energy between the effective potential at some point in space a fixed distance normal to the surface and the Fermi energy. Although it is rather arbitrarily assigned, as far away as $3 \AA$ from the surface, the local work function varies by as much as $0.6-0.8 \mathrm{eV}$ over an equidistant line above the stepped surfaces of Pt and $\mathrm{Pd}^{23}$ This low-potential path may, therefore, provide a channel for electron emission from stepped surfaces. The (311) surface consists of short (100) terraces, with (111) steps. Although there is a greater concentration of steps than on the $6 \times(001)$ surface, the reduction in work function is less and there is a much smaller increase in electron emission. We speculate that both effects are due to the steps being so close together that the Smoluchowski charge smoothing ${ }^{25}$ overlaps.

The wave-vector-dependence of field emission shows in- teresting differences between the low-index and stepped surfaces. Figure 1 gives the surface tunneling current density as a function of $k_{\|}$, the surface-parallel component of the wave vector-in other words, the integrand of the $k_{\|}$integral in (2). On the low-index surfaces, the current drops off very quickly with increasing $k_{\|}$, and as we expect, the field emission originates from the center of the surface Brillouin zone. On $\operatorname{Pd}(311)$, the current is uniformly stronger than on $\operatorname{Pd}(001)$, but drops off similarly with increasing $k_{\|}$in the direction perpendicular to the steps. For Pd $6 \times(001)$ it is much stronger, and stays strong over the whole range of $k_{\|}$in the narrow surface Brillouin zone in the direction perpendicular to the steps. Pt $6 \times(001)$ is particularly striking, with a peak in current for $k_{\|}$away from the center of the surface Brillouin zone. This corresponds to a peak in the surface density, with $m=0$ angular momentum, at the Fermi energy for this particular wave vector.

We now consider the traditional Fowler-Nordheim plot. Figure 2 shows a plot of $\ln \left(J / F^{2}\right)$ against $(1 / F)$ for field emission from our surfaces. Based on a simplified potential, a plot of $\ln \left(J / F^{2}\right)$ against $(1 / F)$ generally shows a straight line for a small region of applied field strength. ${ }^{26}$ Figure 2 gives a clear demonstration of the linearity of such a plot over a wide range of external field strength $F . \mathrm{Cu}$ and $\mathrm{Au}$ have been shown to exhibit a similar dependence. ${ }^{5}$ However, for modified surfaces (i.e., surfaces involving regions of high aspect ratio, such as carbon nanotube modified surfaces ${ }^{27}$ ), the classical Fowler-Nordheim relation has been shown in some cases to no longer be valid. It has been suggested ${ }^{28}$ that this is because of changes in the shape of the surface-barrier potential, from the form assumed by Fowler-Nordheim. As we would expect, the flat emitter surfaces show a straight Fowler-Nordheim plot in our calculations (Fig. 2). However, what is surprising is that the stepped surface, Pt $6 \times(001)$, also shows linear behavior (Fig. 2), even though the surface potential in this case must be very different from the FowlerNordheim model.

It would be interesting to see the spatially decomposed current density, which can be formulated from (2), but this is left for future work. It would also be useful to find a relation between electron emission and step density. However, present limitations of computer resources prevent calculations on structures larger than those presented here.

We would like to thank our colleague Emyr Macdonald for the provision of computing facilities. Special thanks go to Hiroshi Ishida and his colleagues for providing the embedding and field emission codes, and to Richard Forbes for useful discussions.
${ }^{1}$ A. A. Talin, K. A. Dean, and J. E. Jaskie, Solid-State Electron. 45, 963 (2001).

${ }^{2}$ M. Suzuki, T. Kusunoki, M. Sagawa, and K. Tsuji, IEEE Trans. Electron Devices 49, 1005 (2002).

${ }^{3}$ P. R. Schwoebel and I. Brodie, J. Vac. Sci. Technol. B 13, 1391 (1995).
${ }^{4}$ E. W. Plummer and J. W. Gadzuk, Phys. Rev. Lett. 25, 1493 (1970).

${ }^{5}$ T. Ohwaki, H. Ishida, and A. Liebsch, Phys. Rev. B 68, 155422 (2003).

${ }^{6}$ H. J. Kreuzer, L. C. Wang, and N. D. Lang, Phys. Rev. B 45, 12050 (1992). 
${ }^{7}$ Y. Gohda and S. Watanabe, Phys. Rev. Lett. 87, 177601 (2001)

${ }^{8}$ Y. Gotoh, M. Nagao, D. Nozaki, K. Utsumi, K. Inoue, T. Nakatani, T. Sakashita, K. Betsui, H. Tsuji, and J. Ishikawa, J. Appl. Phys. 95, 1537 (2004).

${ }^{9}$ X. Zheng, G. Chen, Z. Li, S. Deng, and N. Xu, Phys. Rev. Lett. 92, 106803 (2004).

${ }^{10}$ T. Sakata, M. Masutani, and A. Sakai, Surf. Sci. 542, 205 (2003).

${ }^{11}$ S. Kitami, T. Nakane, A. Sakai, and T. Sakata, Ultramicroscopy 73, 37 (1998).

${ }^{12}$ R. Ishii, K. Matsumura, A. Sakai, and T. Sakata, Appl. Surf. Sci. 169-170, 658 (2001).

${ }^{13}$ J. E. Inglesfield, J. Phys. C 14, 3795 (1981).

${ }^{14}$ H. Ishida, Phys. Rev. B 63, 165409 (2001).

${ }^{15}$ M. Büttiker, Phys. Rev. Lett. 57, 1761 (1986).

${ }^{16}$ D. Wortmann, H. Ishida, and S. Blügel, Phys. Rev. B 66, 075113 (2002).

${ }^{17}$ A. P. J. Stampfl, R. Martin, P. Gardner, and A. M. Bradshaw, Phys. Rev. B 51, 10197 (1995).

${ }^{18}$ N. V. Smith, C. T. Chen, and M. Weinert, Phys. Rev. B 40, 7565
(1989).

${ }^{19}$ S. L. Hulbert, P. D. Johnson, and M. Weinert, Phys. Rev. B 34, 3670 (1986).

${ }^{20}$ N. Memmel and E. Bertel, Phys. Rev. Lett. 75, 485 (1995).

${ }^{21}$ H. Ishida, D. Wortmann, and T. Ohwaki, Phys. Rev. B 70, 085409 (2004).

${ }^{22}$ A. L. Yeyati and M. Büttiker, Phys. Rev. B 52, R14360 (1995).

${ }^{23}$ I. Merrick, J. E. Inglesfield, and G. A. Attard, Phys. Rev. B 71, 085407 (2005).

${ }^{24}$ K. Wandelt, Appl. Surf. Sci. 111, 1 (1997).

${ }^{25}$ R. Smoluchowski, Phys. Rev. 60, 661 (1941).

${ }^{26}$ A. Modinos, Field, Thermionic and Secondary Electron Emission Spectroscopy (Plenum Press, New York, 1984).

${ }^{27}$ P. G. Collins and A. Zettl, Phys. Rev. B 55, 9391 (1997).

${ }^{28}$ D. P. Woodruff and T. A. Delchar, Modern Techniques of Surface Science, 2nd ed. (Cambridge University Press, Cambridge, England, 1994). 